\title{
Bovine immunoglobulin concentrate-Clostridium difficile retains $C$ difficile toxin neutralising activity after passage through the human stomach and small intestine
}

M Warny, A Fatimi, E F Bostwick, D C Laine, F Lebel, J T LaMont, C Pothoulakis, C P Kelly

Gastroenterology Division, Beth Israel Deaconess Medical Centre, Harvard Medical School, Boston, Massachusetts, USA

M Warny

A Fatimi

J T LaMont

C Pothoulakis

C P Kelly

GalaGen Inc., Arden Hills, Minnesota, USA

E F Bostwick

D C Laine

F Lebel

Correspondence to: Dr C P Kelly, Dana 601, Gastroenterology, Beth Israe Deaconess Medical Centre, 330 Brookline Avenue, Boston, MA 02215, USA.

Accepted for publication 19 August 1998

\begin{abstract}
Background-Bovine immunoglobulin concentrate (BIC)-Clostridium difficile is prepared from the colostrum of cows immunised against $C$ difficile toxins and contains high concentrations of neutralising IgG antitoxin.

Aims-To determine the proportion of BIC-C difficile which survives passage through the human stomach and small intestine.

Methods-Six volunteers with an end ileostomy took $5 \mathrm{~g}$ of BIC- $C$ difficile containing $2.1 \mathrm{~g}$ of bovine IgG on four occasions: alone, with an antacid, during treatment with omeprazole, and within enteric coated capsules.

Results-When BIC-C difficile was taken alone, a mean (SEM) of 1033 (232) $\mathrm{mg}$ of bovine IgG was recovered in the ileal fluid representing $49 \%$ of the total ingested dose. Bovine IgG recovery was not significantly increased by antacid (636 (129) $\mathrm{mg}$ ) or omeprazole (1052 (268) $\mathrm{mg})$. The enteric capsules frequently remained intact or only partially opened in the ileal effluent and free bovine IgG levels were low in this treatment group (89 (101) $\mathrm{mg}$ ). Bovine IgG recovery was higher in volunteers with shorter (less than two hours) mouth to ileum transit times $(68 \%$ versus $36 \%, p<0.05)$. Specific bovine IgG against $C$ difficile toxin $\mathrm{A}$ was detected in ileal fluid following oral BIC. Toxin neutralising activity was also present and correlated closely with bovine IgG levels $(r=0.95, \mathrm{p}<0.001)$.

Conclusion-BIC- $C$ difficile resists digestion in the human upper gastrointestinal tract and specific anti- $C$ difficile toxin $A$ binding and neutralising activity was retained. Passive oral immunotherapy with anti- $C$ difficile BIC may be a useful non-antibiotic approach to the prevention and treatment of $C$ difficile antibiotic associated diarrhoea and colitis.

(Gut 1999;44:212-217)
\end{abstract}

Keywords: pseudomembranous colitis; toxin; diarrhoea; IgG; immunotherapy; antibiotic; Clostridium difficile

Clostridium difficile infection is a common cause of diarrhoea in hospital and nursing home patients. ${ }^{12}$ Antibiotic therapy alters the normal colonic microflora and allows opportunistic infection by $C$ difficile. ${ }^{1-3}$ Infection is more likely to occur in hospital than in outpatients receiving antibiotics because hospital patients and their environment frequently harbour $C$ difficile and its spores. $C$ difficile diarrhoea and colitis are caused by two protein exotoxins produced by pathogenic strains of the organism. ${ }^{3}$ Toxin A is a $308 \mathrm{kDa}$ protein with cytotoxic, enterotoxic, and proinflammatory effects. Toxin $\mathrm{B}$, a $280 \mathrm{kDa}$ protein, is a more potent cytotoxin than toxin $\mathrm{A}$, but is not enterotoxic for rodent intestine. However, toxin B does cause injury to human colonic mucosa in vitro. ${ }^{4}$ Toxin $\mathrm{A}$ and toxin $\mathrm{B}$ show considerable sequence homology and both share the same intracellular mechanism of cytotoxicity. They catalyse the monoglucosylation of small GTP binding rho proteins leading to disruption of the cytoskeleton, cell rounding, and cell death. ${ }^{56}$

$C$ difficile colitis is currently treated by antimicrobial therapy using metronidazole or vancomycin. ${ }^{12}$ These agents are effective in the short term. However, when treatment is discontinued up to $20 \%$ of patients suffer a recurrence of diarrhoea and, in some instances, multiple recurrences occur requiring repeated courses of therapy. Treatment with metronidazole or vancomycin may predispose to recurrent $C$ difficile diarrhoea by causing further disruption of the colonic microflora, a primary event in the pathogenesis of $C$ difficile colitis. ${ }^{7}$ The use of antimicrobial agents to treat antibiotic associated diarrhoea has also led to concern regarding nosocomial bacterial antibiotic resistance; in particular vancomycin resistance in enterococcal species. ${ }^{18}$ These issues have led to a search for an effective, nonantibiotic agent, for prevention or treatment of nosocomial $C$ difficile diarrhoea.

We previously reported the production of a hyperimmune bovine colostral antibody preparation against $C$ difficile. ${ }^{9-11}$ Holstein cows are immunised against $C$ difficile and its toxins during the gestation period and later an immunoglobulin concentrate is prepared from their colostrum. The bovine immunoglobulin concentrate-Clostridium difficile (BIC-C difficile) contains high levels of IgG against $C$ difficile toxins, neutralises the biological effects of $C$ difficile toxins in vitro and, in animal studies,

Abbreviations used in this paper: BIC, bovine immunoglobulin concentrate. 


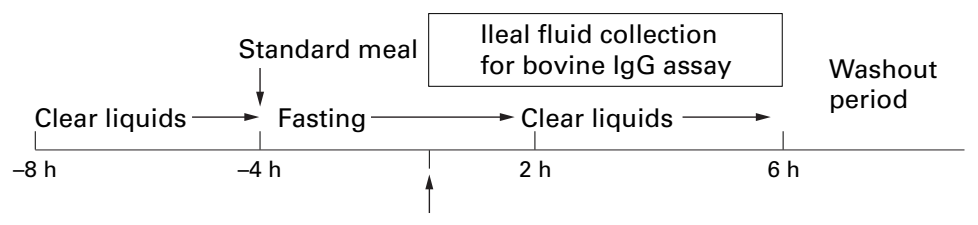

BIC $C$ difficile administered orally

1. BIC C difficile

2. BIC C difficile with antacid

3. BIC C difficile during therapy with omeprazole

4. BIC C difficile in enteric coated capsules

Figure 1 Outline of clinical study protocol.

protects against $C$ difficile toxin induced enterocolitis. ${ }^{9-11}$ BIC- $C$ difficile is designed for use as an oral passive immunotherapy against $C$ difficile and its toxins. To be therapeutically active, oral BIC-C difficile must exert toxin neutralising activity in the human colon. In an earlier study of healthy volunteers, we found detectable levels of bovine IgG as well as $C$ difficile toxin neutralising activity in the stool after oral BIC- $C$ difficile administration. ${ }^{11}$ In that study protection from acidic gastric secretions and short gastrointestinal transit times both appeared to increase faecal bovine IgG concentrations.

The survival of intact bovine IgG in the faeces is relevant to the therapeutic use of BIC- $C$ difficile. However, of equal or even greater importance, is the delivery of intact bovine IgG to the human colon. In this study, volunteers with an end ileostomy but otherwise apparently normal gastrointestinal function took BIC-C difficile orally and their ileal fluid effluent was collected for analysis. The main study aims were to measure accurately the amount of bovine IgG surviving passage to the distal small intestine in humans and to determine whether specific $C$ difficile toxin binding and neutralising activity was preserved. Additional aims were to determine whether reduced exposure to acidic gastric secretions or individual variations in upper gastrointestinal transit times would significantly alter bovine IgG survival.

\section{Methods}

PREPARATION OF BOVINE IMMUNOGLOBULIN

CONCENTRATE-CLOSTRIDIUM DIFFICILE

BIC- $C$ difficile was prepared from the colostral milk of Holstein cows, as previously described. ${ }^{9-11}$ For this study, cows were immunised with a toxoid of purified Clostridium difficile toxin $\mathrm{A}$. The resulting BIC- $C$ difficile contained $42 \mathrm{mg}$ of bovine IgG per $100 \mathrm{mg}$ of powdered concentrate. This preparation of BIC- $C$ difficile showed high concentrations of anti- $C$ difficile toxin $\mathrm{A}$ activity as demonstrated by: (1) ELISA; (2) inhibition of toxin A binding to its brush border membrane receptor; (3) neutralisation of the cytotoxic effects of toxin $\mathrm{A}$ in the tissue culture cytotoxicity assay; and (4) inhibition of the enterotoxic effects of toxin $\mathrm{A}$ in the rodent ileal loop assay. ${ }^{10}$

HUMAN STUDY PROTOCOL

A single site, open, phase I study was performed in volunteers with an end ileostomy to examine the safety and bioavailability of a single oral dose of BIC-C difficile. The study protocol was approved by the Beth Israel Deaconess Medical Centre Committee on Clinical Investigations. An outline of the study protocol is illustrated in fig 1 .

\section{Study subjects}

Six volunteers (four men, two women; mean age 49 years, range 35-69) with a surgically created end ileostomy were enrolled into the study. In all cases, end ileostomy was performed following colectomy. The indications for colectomy were ulcerative colitis in five patients and indeterminate colitis in one. At study enrolment none of the patients had a history or any clinical evidence of upper gastrointestinal tract disease apart from ileostomy. All volunteers reported stable ileostomy function during the six month period prior to study entry.

Exclusion criteria included: a history of other gastrointestinal diseases or surgery which might be expected to affect normal gastrointestinal function, a history of clinically significant cow's milk protein allergy or other allergy to milk products, lactose intolerance, evidence of ongoing systemic or infectious disease, or use of medication that might be expected to affect normal gastrointestinal function. All volunteers received monetary compensation for study participation.

\section{Administration of BIC-C difficile}

BIC- $C$ difficile $5 \mathrm{~g}$, containing $2.1 \mathrm{~g}$ of bovine IgG, was administered orally. Volunteers adhered to a clear liquid diet from eight hours until four hours prior to BIC- $C$ difficile administration at which time they consumed a standard meal (fig 1). They then fasted for four hours after which the BIC- $C$ difficile was consumed, reconstituted in $250 \mathrm{ml}$ of an isosmotic polyethylene glycol solution containing $421 \mathrm{mg}$ of sodium bicarbonate. The polyethylene glycol was used as a nonabsorbable transit marker. After a further two hours they were again allowed to take clear liquids until the end of the six hour ileostomy fluid collection period. Each volunteer was studied on four separate occasions with an intervening washout period of at least 72 hours. The BIC-C difficile was taken alone, with an antacid (30 $\mathrm{ml}$ of BICTRA), during therapy with the proton pump inhibitor omeprazole (Prilosec $20 \mathrm{mg}$, twice daily for four days prior to and on the day of BIC ingestion), or within enteric coated capsules. Treatments were administered in random order except omeprazole which was given last to avoid any carryover effect. The enteric coated capsules were designed to increase colonic delivery of BIC- $C$ difficile and were prepared by the Pharmaceutical Service Division, College of Pharmacy, University of Iowa, as described previously. ${ }^{11}$ Twelve hard gelatin capsules were consumed, each containing $420 \mathrm{mg}$ of BIC and coated with two layers of cellulose acetate phthalate.

Collection of ileostomy fluid

Fluid was completely drained from the volunteers' ileostomy appliance immediately prior to 
BIC- $C$ difficile ingestion and, at 30 minute intervals, for six hours thereafter. The fluid volume was recorded and an aliquot taken which was filter sterilised and used for cytotoxicity neutralisation experiments. Protease inhibitors were added to the remaining fluid which was immediately frozen and stored at $-20^{\circ} \mathrm{C}$. The final concentrations of protease inhibitors were as follows: aprotinin $10 \mu \mathrm{g} / \mathrm{ml}$, leupeptin $10 \mu \mathrm{g} / \mathrm{ml}$, phenylmethylsulphonyl fluoride $10 \mu \mathrm{g} / \mathrm{ml}, \mathrm{N}$ - $p$-tosyl-L-lysine chloromethyl ketone $10 \mu \mathrm{g} / \mathrm{ml}$, and L-1-tosylamido-2phenylethyl chloromethyl ketone $10 \mu \mathrm{g} / \mathrm{ml}$.

SPECIAL LABORATORY STUDIES

Total bovine IgG levels in the ileostomy fluid were measured by single radial immunodiffusion. ${ }^{10}{ }^{11}$ Specific anti- $C$ difficile toxin A bovine $\operatorname{IgG}$ levels were measured by enzyme linked immunosorbent assay (ELISA) as previously described. ${ }^{10}{ }^{11}$ Cytotoxicity was determined by rounding of Chinese hamster ovary (CHO) cells (American Type Culture Collection, Rockville, Maryland, USA) in monolayer culture after exposure to purified $C$ difficile toxin $\mathrm{A}$. The minimum $50 \%$ cytotoxic dose of toxin A was defined as the minimum dose resulting in $50 \%$ cell rounding at 24 hours $(40 \mathrm{ng} / \mathrm{ml}$ in these experiments). Inhibition of cytotoxicity was quantified by adding serial twofold dilutions of the ileostomy fluid to purified $C$ difficile toxin A to achieve an end concentration of twice the minimum 50\% cytotoxic dose of toxin A. After 30 minutes the mixture was added to the cell monolayers and cell rounding was assessed after 24 hours as previously described. ${ }^{1011}$

STATISTICAL ANALYSES

Statistical analyses were performed using SigmaStat for Windows version 1.00 (Jandel Scientific Software, San Rafael, California, USA). Unless stated otherwise, analysis of variance was used for intergroup comparisons and the Spearman rank order test was used to evaluate correlations.

\section{Results}

STUDY SUBJECTS AND SAMPLE COLLECTION

Six volunteers participated in the study and all completed the study protocol. However, during the study one volunteer developed symptoms of ileostomy dysfunction and, two weeks after study termination, required hospital admission because of small bowel obstruction. This resolved without surgical intervention. Ileostomy samples from this volunteer were excluded from the data analysis. No other sig-

Table 1 Time of peak bovine IgG recovery from ileal fluid following administration of oral bovine immunoglobulin concentrate- $C$ difficile

\begin{tabular}{lllll}
\hline Subject & Alone & Antacid & Omeprazole & Capsules \\
\hline 1 & $60 \mathrm{~min}(1632 \mathrm{mg})$ & $90 \mathrm{~min}(963 \mathrm{mg})$ & $90 \mathrm{~min}(901 \mathrm{mg})$ & $360 \mathrm{~min}(118 \mathrm{mg})$ \\
2 & $60 \mathrm{~min}(1558 \mathrm{mg})$ & $60 \mathrm{~min}(811 \mathrm{mg})$ & $60 \mathrm{~min}(1396 \mathrm{mg})$ & $300 \mathrm{~min}(57 \mathrm{mg})$ \\
3 & $150 \mathrm{~min}(611 \mathrm{mg})$ & $150 \mathrm{~min}(677 \mathrm{mg})$ & $210 \mathrm{~min}(1275 \mathrm{mg})$ & $360 \mathrm{~min}(244 \mathrm{mg})$ \\
4 & $150 \mathrm{~min}(596 \mathrm{mg})$ & $180 \mathrm{~min}(195 \mathrm{mg})$ & $150 \mathrm{~min}(622 \mathrm{mg})$ & $240 \mathrm{~min}(26 \mathrm{mg})$ \\
5 & $150 \mathrm{~min}(767 \mathrm{mg})$ & $120 \mathrm{~min}(534 \mathrm{mg})$ & $180 \mathrm{~min}(1067 \mathrm{mg})$ & $(0 \mathrm{mg})$ \\
\hline
\end{tabular}

The collection time point at which ileal fluid contained the greatest amount of bovine IgG is shown for each subject and each study arm. The total amounts of free bovine IgG recovered during each six hour collection period are also shown (in parentheses).

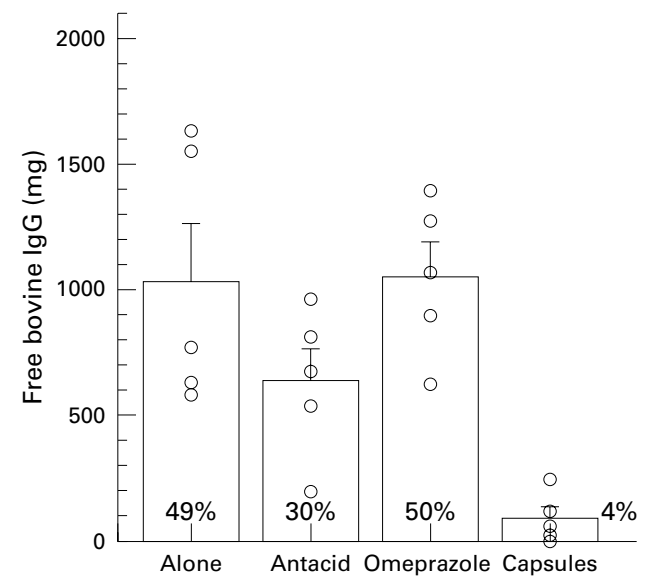

Figure 2 Recovery of free bovine IgG from the ileum after oral administration of BIC-C difficile. Results expressed as mean (SEM); individual data points are shown. The mean recovery expressed as a percentage of the total amount of ingested bovine IgG is also shown.

nificant adverse experiences were encountered by the study volunteers.

ILEAL RECOVERY OF BOVINE IgG AFTER ORAL BIC-C DIFFICILE

Figure 2 shows the total free bovine IgG content of the six hour ileostomy fluid collections. When the BIC- $C$ difficile was taken alone the mean (SEM) recovery of bovine IgG from the terminal ileum was 1033 (232) mg. This represented $49 \%$ of the total ingested dose of $2100 \mathrm{mg}$ of bovine IgG. The total ileal recovery of bovine IgG was 636 (129) $\mathrm{mg} \mathrm{(30 \%}$ of the oral dose) when additional acid buffering capacity was provided in the form of an oral antacid. The omeprazole group had the highest ileal bovine IgG content at 1052 (268) mg ( $50 \%$ of the oral dose). However, analysis of variance did not reveal any significant difference between these three treatment groups $(p=0.13)$.

The mean recovery of free bovine IgG from the ileal fluid after ingestion of BIC- $C$ difficile in enteric coated capsules was 89 (45) $\mathrm{mg}$ (4\% of the oral dose). This was significantly less than for the BIC- $C$ difficile alone and omeprazole groups $(\mathrm{p}<0.05$ for both). However, many of the capsules were recovered intact or only partially opened. Furthermore, when free bovine IgG was detected it was only found at the latter end of the six hour collection period (table 1). For all other treatment groups peak bovine IgG levels were detected much earlier (table 1) and negligible amounts of bovine IgG were found at six hours (fig 3). Since the release of bovine IgG was delayed and a substantial amount remained encapsulated the measurement of free bovine IgG in the ileal fluid is an incomplete measure of BIC- $C$ difficile survival in this treatment group.

TIME COURSE OF BOVINE IgG TRANSIT THROUGH THE UPPER GASTROINTESTINAL TRACT Figure 3 depicts the time course of bovine IgG recovery from ileal fluid. Bovine IgG first reached the distal ileum 30 to 60 minutes after oral administration of reconstituted BIC- $C$ dif- 


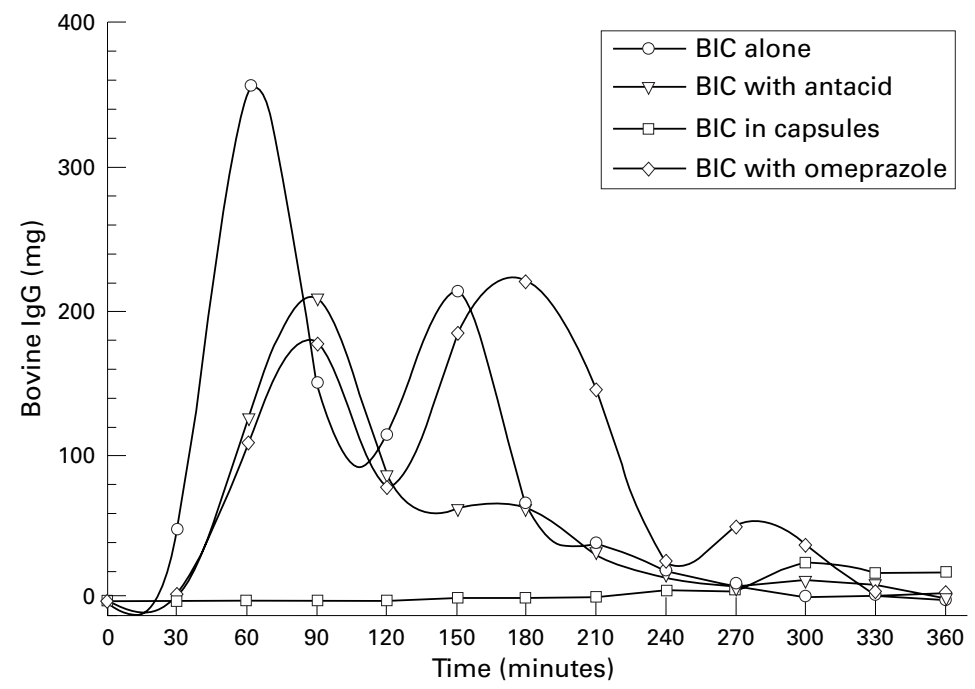

Figure 3 Time course of bovine IgG recovery from the ileum after oral BIC-C difficile. The mean amounts of bovine IgG recovered at each collection time are shown for each treatment group $(n=5)$.

ficile solution. The highest bovine IgG concentrations were observed between 60 and 180 minutes. Very little IgG was recovered after 300 minutes. In Figure 3, a biphasic pattern is evident with two peaks of bovine IgG concentrations: the first at 60 to 90 minutes and the second at 150 to 180 minutes after ingestion. In fact, this reflects two groups of subjects with different upper gastrointestinal tract transit times rather than a biphasic delivery of IgG to the distal small intestine. As shown in table 1, two subjects ( 1 and 2 ) had consistently shorter transit times causing the early peak at 60 to 90 minutes. The remaining three subjects had slower transit times and these accounted for the second peak in mean bovine IgG concentration at 150 to 180 minutes. Release of free bovine IgG from enteric coated capsules was limited and delayed.

When the three groups receiving reconstituted BIC were examined, the average recovery of bovine $\mathrm{IgG}$ was twofold higher in the two subjects (1 and 2) with short transit times (1421 $\mathrm{mg} ; 68 \%$ of the total dose) when compared with the three subjects with longer transit times $(705 \mathrm{mg} ; 36 \%$ of the total dose; $\mathrm{p}=0.047$ by paired $t$ test).
ILEAL FLUID C DIFFICILE TOXIN A BINDING AND NEUTRALISING ACTIVITY

Having determined that approximately $50 \%$ of the total bovine IgG from oral BIC-C difficile survived passage to the distal small intestine, we next examined whether specific anti- $C$ difficile toxin activity was retained. Binding of bovine IgG to purified $C$ difficile toxin A was determined by ELISA. A positive correlation was evident between the specific bovine IgG anti- $C$ difficile toxin $\mathrm{A}$ activity of each ileal fluid sample and its total bovine IgG content $(r=$ $0.84, \mathrm{p}<0.001, \mathrm{n}=197)$.

Figure $3 \mathrm{~A}$ illustrates the total bovine $\mathrm{IgG}$ and specific toxin A binding activity of ileal fluid collected from subject 2 after ingestion of BIC- $C$ difficile. This subject had a relatively short transit time (60 minutes to peak ileal fluid bovine IgG level) and high total bovine IgG recovery ( $74 \%$ of the total dose). Figure $4 \mathrm{~B}$ illustrates the total bovine IgG and specific toxin A binding activity of ileal fluid collected from subject 3 after ingestion of BIC- $C$ difficile. This subject had a longer transit time (150 minutes to peak ileal fluid bovine IgG level) and a lower total bovine IgG recovery (29\% of the total dose). In both instances, the pattern of specific antitoxin A activity closely paralleled the bovine IgG concentrations.

The ability of ileal fluid samples to neutralise the biological activity of $C$ difficile toxin A was then determined using the tissue culture cytotoxicity assay. Ileal fluid samples containing high levels of bovine IgG showed toxin neutralising effect whereas those with no measurable bovine IgG had little if any toxin neutralising activity. Negative control ileostomy fluid, obtained from study subjects immediately prior to BIC- $C$ difficile ingestion, showed no detectable toxin neutralising activity. When the toxin A neutralising titre of a range of ileal fluid samples was determined, a positive correlation between neutralising titre and ileal fluid bovine IgG level was confirmed $(r=0.95, \mathrm{p}<0.001$, $\mathrm{n}=24$ ) (fig 5).

\section{Discussion}

The main finding of this study is that almost half of the bovine IgG in an oral dose of BIC-C difficile can be retrieved intact from the distal small intestine in humans. The surviving IgG
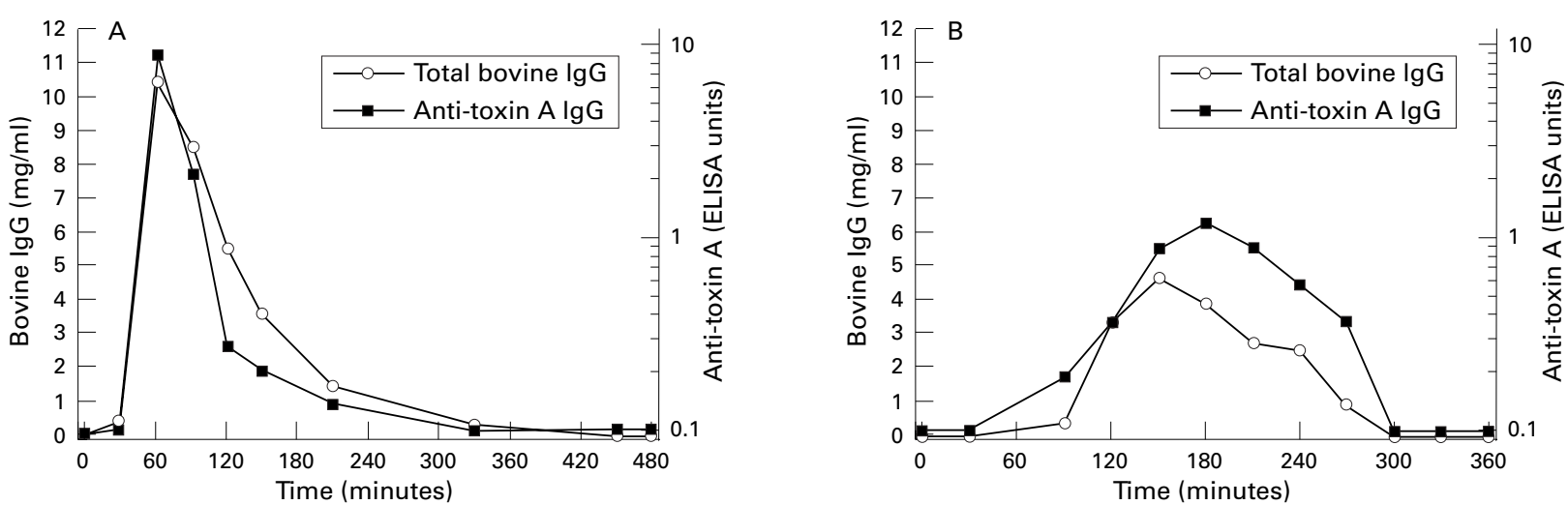

Figure 4 Comparison of specific anti-C difficile toxin $A$ and total bovine IgG levels in ileal fluid after oral BIC-C difficile. (A) Levels for subject 2 , receiving BIC-C difficile alone. Total recovery was $1558 \mathrm{mg}$ or $74 \%$ of the ingested dose of bovine IgG. (B) Levels for subject 3, receiving BIC-C difficile alone. Total recovery was $611 \mathrm{mg}$ or $29 \%$ of the ingested dose of bovine IgG. 


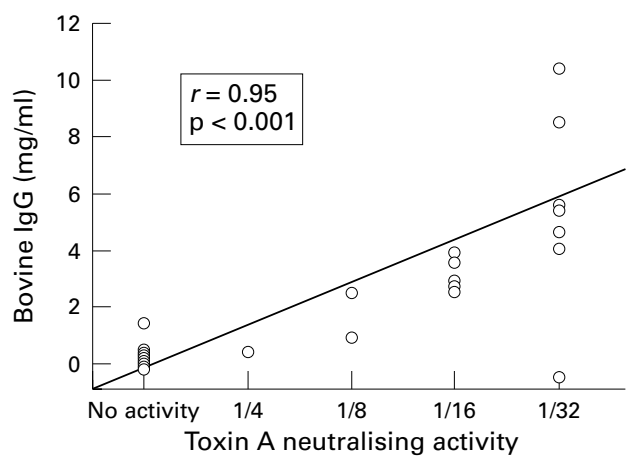

Figure 5 Comparison of specific anti-C difficile toxin $A$ neutralising activity and total bovine IgG levels in ileal fluid after oral BIC-C difficile. The greatest dilution of ileal fluid which neutralised toxin $A$ is shown together with the bovine IgG level of that ileal fluid sample.

retains its specific antigen binding activity, in this instance binding to and neutralising $C$ difficile toxin A. This finding supports the feasibility of using BIC as oral passive immunotherapy against enteric pathogens. ${ }^{12-17}$ Oral administration of BIC results in substantial levels of bovine IgG within the lumen of the human small intestine. Thus, infectious diarrhoea caused by small bowel pathogens such as Cryptosporidium parvum, microsporidial species, or Vibrio cholerae may be amenable to prophylaxis or therapy using appropriate hyperimmune bovine colostral antibody preparations. ${ }^{12} 131718$ Our study findings also suggest that BIC may be effective in colonic infectious diarrhoea since a substantial portion of the oral dose of bovine IgG is delivered intact to the caecum. ${ }^{14-16}$

Roos et al administered a ${ }^{15} \mathrm{~N}$-labelled preparation of bovine immunoglobulin concentrate to volunteers and sampled their ileal content using a nasointestinal tube. ${ }^{19}$ They calculated a $19 \%$ recovery rate for intact ${ }^{15} \mathrm{~N}-\mathrm{IgG}$ in the ileum, substantially lower that the $49 \%$ recovered in this study. In an earlier study, we found that exposure of BIC- $C$ difficile to acidic human gastric secretions $(\mathrm{pH}<4)$ resulted in rapid loss of bovine IgG activity. ${ }^{11}$ In this study, the BIC- $C$ difficile was administered in a solution containing sodium bicarbonate. The additional buffering capacity of this solution may have improved bovine IgG survival in the stomach, thereby increasing ileal recovery of intact $\mathrm{IgG}$. The use of additional antacid or a proton pump inhibitor did not result in any further significant increase in bovine IgG survival. Thus, it appears unnecessary to use these extra measures to protect oral BIC-C difficile from gastric acid degradation.

In our earlier study, we also found that faecal bovine IgG levels are notably increased when oral BIC-C difficile is administered within enteric coated capsules. ${ }^{11}$ Our previous interpretation of this finding was that the enteric capsules protected BIC from gastric acid degradation. The findings of this study suggest an additional explanation. The capsules frequently passed through the stomach and small intestine intact. When bovine IgG was released from the capsules, this typically occurred at the latter end of the six hour ileo- stomy fluid collection. These findings suggest that enteric capsules may protect against bovine IgG degradation by delaying the release of BIC rather than by acid protection alone. This interpretation is further supported by our finding that volunteers with slower mouth to ileum transit times had significantly lower bovine IgG recovery than those with more rapid transit. For example, when BIC- $C$ difficile was taken alone $76 \%$ of the oral dose was recovered from the distal ileum in volunteers with transit times of less than two hours whereas only $31 \%$ was recovered from those with transit times of more than two hours. A prolonged gastrointestinal transit time was also found to reduce bovine IgG recovery from the stool. ${ }^{11}$ Thus, it appears that enteric encapsulation raises faecal bovine IgG levels by delaying the release of BIC until the distal small intestine or colon. Formulation of BIC- $C$ difficile for targeted release in the colon may increase its efficacy in neutralising $C$ difficile toxins within the colonic lumen and at the same time allow a reduction in the oral dose.

Animals immunised against $C$ difficile toxins are protected against challenge with toxigenic $C$ difficile whereas non-immunised animals develop fatal enterocolitis. ${ }^{20-22}$ Some, but not all, clinical studies indicate that patients with severe or recurrent $C$ difficile colitis have an inadequate antibody response to $C$ difficile and its toxins. ${ }^{23-28}$ The first animal study describing passive immunotherapy for $C$ difficile enterocolitis was reported in 1979; hamsters were treated with parenteral Clostridium sordellii antitoxin, which cross reacts with $C$ difficile toxins $\mathrm{A}$ and $\mathrm{B}$, and protected against clindamycin induced colitis. ${ }^{29}$ Protective oral immunisation of hamsters using breast milk was reported in $1987 . .^{22}$ There are also limited reports of passive immunotherapy against $C$ difficile diarrhoea and colitis in humans. ${ }^{26}{ }^{30}{ }^{31} \mathrm{~A}$ number of patients with recurrent, or refractory, $C$ difficile diarrhoea have been treated using pooled normal human IgG administered intravenously. ${ }^{26}{ }^{30}$ This treatment increases serum anti- $C$ difficile toxin antibody concentrations and was effective in controlling symptoms in the small number of cases reported. In the present study, we showed that oral administration of BIC- $C$ difficile provides $C$ difficile toxin binding and neutralising activity in the distal ileum in human volunteers. A randomised, controlled, clinical trial examining the efficacy of BIC- $C$ difficile in treating mild to moderately severe $C$ difficile diarrhoea is now underway.

Portions of this study were performed at the Beth Israel Deaconess Medical Centre, General Clinical Research Centre, supported in part by grant RR01032 from the National Institutes of Health.

1 Fekety R. Guidelines for the diagnosis and management of Clostridium difficile-associated diarrhea and colitis. American College of Gastroenterology, Practice Parameters Committee. Am f Gastroenterol 1997;92:739-50.

2 Kelly CP, Pothoulakis C, LaMont JT. Clostridium difficile colitis. N Engl f Med 1994;330:257-62.

3 Pothoulakis C. Pathogenesis of Clostridium difficileassociated diarrhoea. Eur $\mathcal{F}$ Gastroenterol Hepatol 1996;8: 1041-7.

4 Riegler M, Sedivy R, Pothoulakis C, et al. Clostridium difficile toxin $\mathrm{B}$ is more potent than toxin $\mathrm{A}$ in damaging human colonic epithelium in vitro. 7 Clin Invest 1995;95: 2004-11. 
5 Just I, Wilm M, Selzer J, et al. The enterotoxin from Clostridium difficile (ToxA) monoglucosylates the Rho proteins. F Biol Chem 1995;270:13932-6.

6 Just I, Selzer J, Wilm M, et al. Glucosylation of Rho protein by Clostridium difficile toxin B. Nature 1995;375:500-3.

7 Borriello SP. The influence of the normal flora on Clostrid ium difficile colonisation of the gut. Ann Med 1990;22:617.

8 Linevsky JK, Kelly CP. Clostridium difficile colitis. In: LaMont JT, ed. Gastrointestinal infections: diagnosis and management. New York: Marcel Dekker, 1997:293-325.

9 Lyerly DM, Bostwick EF, Binion SB, et al. Passive immunization of hamsters against disease caused by Clostridium difficile by use of bovine immunoglobulin $\mathrm{G}$ concentrate. Infect Immun 1991;59:2215-18.

10 Kelly CP, Pothoulakis C, Vavva F, et al. Anti-Clostridium difficile bovine immunoglobulin concentrate inhibits cytotoxicity and enterotoxicity of C difficile toxins. Antimicrob Agents Chemother 1996;40:373-9.

11 Kelly CP, Chetham S, Keates S, et al. Survival of anti-Clostridium difficile bovine immunoglobulin concenanti-Clostridium difficile bovine immunoglobulin concentrate in the human gastroint

12 Nord J, Ma P, DiJohn D, et al. Treatment with bovine hyperimmune colostrum of cryptosporidial diarrhea in AIDS patients. AIDS 1990;4:581-4.

13 Davidson GP, Whyte PB, Daniels E, et al. Passive immunisation of children with bovine colostrum containing antibodies to human rotavirus. Lancet 1989;ii:709-12.

14 Tacket CO, Losonsky G, Link H, et al. Protection by milk immunoglobulin concentrate against oral challenge with enterotoxigenic Escherichia coli. $N$ Engl f Med 1988;318: 1240-3.

15 Tacket CO, Binion SB, Bostwick E, et al. Efficacy of bovine milk immunoglobulin concentrate in preventing illness after Shigella flexneri challenge. Am f Trop Med Hyg 1992; 47:276-83.

16 Mietens C, Keinhorst H, Hilpert H, et al. Treatment of infantile E. coli gastroenteritis with specific bovine anti-E. infantile E. coli gastroenteritis with specific bovine anti-E. 52 .

17 Brunser O, Espinoza J, Figueroa G, et al. Field trial of an infant formula containing anti-rotavirus and antiEscherichia coli milk antibodies from hyperimmunized cows. F Pediatr Gastroenterol Nutr 1992;15:63-72.
18 Brussow H, Hilpert H, Walther I, et al. Bovine milk immunoglobulins for passive immunity to infantile rotavirus gasnoglobulins for passive immunity to infantile
troenteritis. $\mathcal{F}$ Clin Microbiol 1987;25:982-6.

19 Roos N, Mahe S, Benamouzig R, et al. ${ }^{15} \mathrm{~N}$-labeled immunoglobulins from bovine colostrum are partially resistant to digestion in human intestine. 7 Nutr 1995;125: $1238-44$.

20 Libby JM, Jortner BS, Wilkins TD. Effects of the two toxins of Clostridium difficile in antibiotic-associated cecitis in hamsters. Infect Immun 1982;36:822-9.

21 Fernie DS, Thomson RO, Batty I, et al. Active and passive immunization to protect against antibiotic associated caecitis in hamsters. Dev Biol Stand 1983;53:325-32.

$22 \mathrm{Kim}$ PH, Iaconis JP, Rolfe RD. Immunization of adult hamsters against Clostridium difficile-associated ileocecitis and transfer of protection to infant hamsters. Infect Immun 1987;55:2984-92.

23 Aronsson B, Granstrom M, Mollby R, et al. Serum antibody response to Clostridium difficile toxins in patients with Clostridium difficile diarrhoea. Infection 1985;13:97-101.

24 Johnson S, Gerding DN, Janoff EN. Systemic and mucosal antibody responses to toxin A in patients infected with antibody responses to toxin A in patients infected

25 Kelly CP. Immune response to Clostridium difficile infection. Eur f Gastroenterol Hepatol 1996;8:1048-53.

26 Leung DY, Kelly CP, Boguniewicz M, et al. Treatment with intravenously administered gamma globulin of chronic relapsing colitis induced by Clostridium difficile toxin. $\mathcal{F}$ Pediatr 1991;118:633-7.

27 Mulligan ME, Miller SD, McFarland LV, et al. Elevated levels of serum immunoglobulins in asymptomatic carriers of Clostridium difficile. Clin Infect Dis 1993;16(suppl 4): S239-44.

28 Warny M, Vaerman JP, Avesani V, et al. Human antibody response to Clostridium difficile toxin $\mathrm{A}$ in relation to clinical course of infection. Infect Immun 1994;62:384-9.

29 Allo M, Silva J, Jr, Fekety R, et al. Prevention of clindamycin-induced colitis in hamsters by Clostridium clindamycin-induced colitis in hamsters by Clostrii antitoxin. Gastroenterology 1979;76:351-5.

30 sordelli1 antitoxin. Gastroenterology 1979;76:351-5. noglobulin therapy for severe Clostridium difficile colitis. Gut 1997;41:366-70.

31 Tjellstrom B, Stenhammar L, Eriksson S, et al. Oral immunoglobulin A supplement in treatment of Clostridium difficile enteritis. Lancet 1993;341:701-2. 